\title{
A 56 yr-old female with chronic cough and persistent infiltration on CT examination
}

\section{Case history}

A 56-yr-old female who had chronic dry cough for 1 yrwas evaluated. She was otherwise healthy and immunocompetent. There was no history of past or present lung disease, smoking habit or recent oral operation.

Physical examination was normal except for poor oral hygiene. She had a blood pressure of $130 / 70 \mathrm{mmHg}$, a respiratory rate of 16 breaths. $\mathrm{min}^{-1}$, a pulse rate of 82 beats. $\mathrm{min}^{-1}$ and her axillar temperature was $36.5^{\circ} \mathrm{C}$. Complete blood count and routine biochemical parameters were normal. Erythrocyte sedimentation rate was $56 \mathrm{~mm} \cdot \mathrm{h}^{-1}$ and her C-reactive protein level was $0.84 \mathrm{mg} \cdot \mathrm{dL}^{-1}\left(<0.5 \mathrm{mg} \cdot \mathrm{dL}^{-1}\right.$ is normal). Sputum was negative for bacteria, acid-fast bacilli and fungi. In addition, cancer cells were not revealed.

Chest radiography was normal. A computed tomography (CT) scan was carried out and is shown in figure 1 .

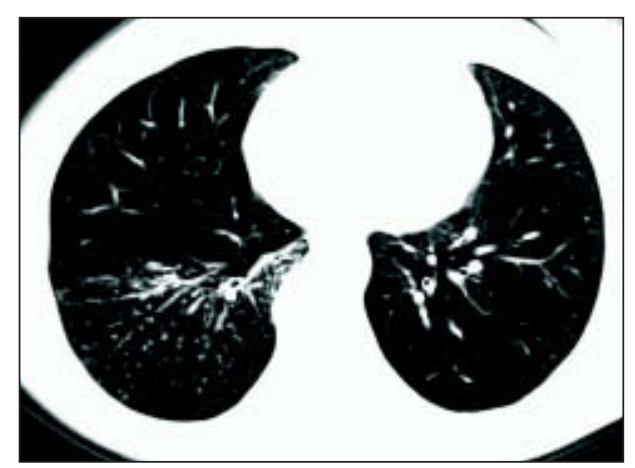

Fig. 1. - Thoracic computed tomography scan

\section{Task 1. \\ Interpret the CT scan}

Because of the clinical and radiological findings, bronchoscopy was applied. Fibreoptic bronchoscopy revealed obstruction of the right lower lobe basal segment orifice with soft tissue mass. Histology of the lesion was obtained by bronchoscopic biopsy (fig. 2).

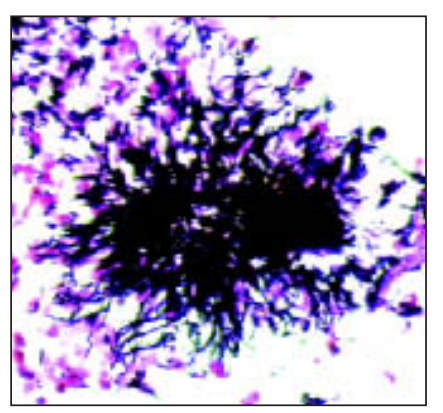

Fig. 2. - Bronchial biopsy specimen (Methenamine silver nitrate staining).

Task 2.

Interpret the pathological results

Task 3.

Suggest a diagnosis

Task 4.

Suggest a treatment
A. Çilli
I. Erogullari
0. Ozbudak
T. Ozdemir

Dept of Pulmonary Diseases, Akdeniz University School of Medicine, Antalya, Turkey.

\section{Correspondence:}

A. Çilli

Dept of Pulmonary Diseases

Akdeniz University Hospital

Dumlupinar Bulvari

Kampus

07058 Antalya

Turkey

Fax: 902422274490

E-mail: acilli@akdeniz.edu.tr 


\section{Interpretation}

\section{Answer 1. \\ Computed tomography}

"The clinical presentation of pulmonary actinomycosis may include cough, fever, expectoration of yellow pus with sulphur granules, recurrent pneumonia, haemoptysis, pleural effusion, and fungus ball-type lesion and medistinal mass."

\section{Discussion}

Actinomyceae is a filamentous, facultative anaerobic bacteria species, which lives in the oropharynx and gastro-intestinal tract. Six out of the fourteen species are pathogenic in humans and the most important pathogen is Actinomyces israelii. There are three classic types of involvement: cervicofacial, abdominopelvic and thoracic [1]. Thoracic involvement is seen in 15-45\% of cases, according to different written sources. Aspiration of organisms from the oropharynx is the usual source of pulmonary actinomyces infection. It may involve the lungs, pleura, mediastinum or chest wall. Poor oral hygiene and associated dental disease may increase the risk of the disease $[1,2]$.

\section{Answer 2. Histopathological results}

Filaments and sulphur granules were apparent in the bronchoscopic biopsy of the mass (fig. 2).

\section{Diagnostic consideration}

In the differential diagnosis of the present case, the following diagnoses were considered:

1) tuberculosis;

2) malignancy;

3) fungal infection; and

4) pulmonary actinomycosis.

\section{Answer 3.} Diagnosis:

Pulmonary actinomycosis.

\section{Answer 4. Treatment}

The patient was treated with intravenous penicillin $G$ (20 million units.day $\left.{ }^{1}\right)$ for 3 weeks in the hospital and then changed to oral amoxicillin (3 g.day').

\section{Clinical course}

After 8 weeks of treatment, cough was almost resolved and a repeat $C T$ of the chest showed reduction in the infiltration.
The clinical presentation of pulmonary actinomycosis may include cough, fever, expectoration of yellow pus with sulphur granules, recurrent pneumonia, haemoptysis, pleural effusion, and fungus ball-type lesion and mediastinal mass. Primary endobronchial actinomycosis is rare and it might be associated with foreign bodies [3]. Endobronchial actinomycosis may manifest as irregular granular thickening and exophytic soft tissue mass with occlusion of bronchi $[1,4]$. Endobronchial presentation might mimic lung cancer, endobronchial lipoma and tuberculosis [5-8]. Endobronchial actinomycosis should be included in the differential diagnosis of any endobronchial mass.

In the patient presented in this image case, the CT scan of the chest showed a "tree-inbud" sign in the right lower lobe. To best of our knowledge, there has been no report of pulmonary actinomycosis being associated with "tree-in-bud" signs in the literature. The "tree-inbud" sign is seen in tuberculosis and it must be considered in the differential diagnosis, especially in regions endemic for tuberculosis. In addition, a "tree-in-bud" sign can be found in bronchopneumonia, diffuse panbronchiolitis, bronchiectasis, cystic fibrosis and allergic bronchopulmonary aspergillosis [9]. The characteristic findings on CT scan in pulmonary actinomycosis include airspace consolidation, mass-like lesions, cavitation and enlargement of mediastinal lymph nodes $[1,2,10,11]$. 
Diagnosis of pulmonary actinomycosis is difficult without surgical intervention. Diagnosis is based on revealing sulphur granules of actinomyces, a positive culture, correlation with the clinical and radiological features, and response to antibiotic therapy. Less than $10 \%$ of cases are diagnosed correctly in the time of admission [2] and the average duration of illness before definitive diagnosis is 6 months [12]. Pulmonary tuberculosis, malignancy, abscesses, vasculitis (Wegener's granulomatosis), empyema and aspergillosis are the most common diseases that should be considered in the differential diagnosis [1, 2].

Actinomycosis is a treatable disease. Penicillin $\mathrm{G}$ is the drug of choice. Large doses
(18-24 million $U$ ) are given daily for 2-6 weeks, followed by $6-12$ months of oral penicillin Vor amoxicillin [1]. Surgical intervention is indicated when a patient has frequent haemoptysis and the lesion does not respond to drug therapy [13]. Central nervous system involvement, onset of the disease over 2 months and lack of antibiotic therapy or surgery are negative prognostic factors associated with poor outcome (death or relapse) $[2,12]$.

In conclusion, pulmonary actinomycosis is a rare disease which has different clinical and radiological characteristics. It should be considered in the differential diagnosis of persistent infiltrative lung lesions.
"Diagnosis of pulmonary actinomycosis is difficult without surgical intervention."

\section{References}

1. Mabeza GF, Macfarlane J. Pulmonary actinomycosis. Eur Respir J 2003; 21: 545-551.

2. Smego RA Jr, Foglia G. Actinomycosis. Clin Infect Dis 1998; 26: 1255-1263.

3. Chouabe S, Perdu D, Deslee G, Milosevic D, Marque E, Lebargy F. Endobronchial actinomycosis associated with foreign body. Chest 2002; 121: 2069-2072.

4. Dujneungkunakorn T, Riantawan P, Tungsagunwattana S. Pulmonary actinomycosis: a study of 16 cases from central chest hospital. J Med Assoc Thai 1999; 82: 531-535.

5. Lau KY. Endobronchial actinomycosis mimicking pulmonary neoplasm. Thorax 1992; 47: 64-65.

6. Ariel I. Endobronchial actinomycosis simulating bronchogenic carcinoma. Chest 1991; 99: 493-495.

7. Jin SL, Lee HP, Kim JI, et al. A case of endobronchial actinomycosis. Korean J Intern Med 2000; 15: 240-244.

8. Lee SH. Endobronchial actinomycosis simulating endobronchial tuberculosis: a case report. J Korean Med Sci 1999; 14: $315-318$.

9. Eisenhuber E. The tree-in-bud sign. Radiology 2002; 222: 771-772.

10. Kwong JS, Muller NL, Godwin JD, et al. Thoracic actinomycosis: CT findings in eight patients. Radiology 1992; 183: 189-192.

11. Conant EF, Wechsler RJ. Actinomycosis and nocardiosis of the lung. J Thorac Imaging 1992; 7: 75-84.

12. Weese WC, Smith IM. A study of 57 cases of actinomycosis over a 36-year period. A diagnostic 'failure' with good prognosis after treatment. Arch Intern Med 1975; 135: 1562-1568.

13. Endo $S$, Murayama F, Yamaguchi $T$, et al. Surgical considerations for pulmonary actinomycosis. Ann Thorac Surg 2002; 74: 185-190. 